\title{
Space Charge Accumulation in Silicone Rubber Influenced by Poole- Frenkel Effect
}

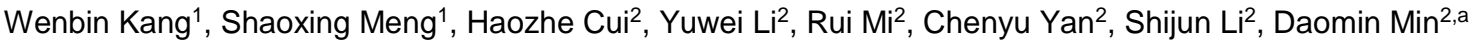 \\ ${ }^{1}$ China Electric Power Research Institute (Wuhan Branch), 430074 Wuhan, Hubei, China \\ ${ }^{2}$ State Key Laboratory of Electrical Insulation and Power Equipment, School of Electrical Engineering, Xi'an Jiaotong University, 710049 \\ Xi'an, Shaanxi, China
}

\begin{abstract}
With the rapid increase of electrical energy consumption in metropolises, more and more power cables are utilized in power grid or urban power network to transmit and distribute electrical energy. Silicone rubber is widely used as polymeric insulating materials of power cable accessories due to their excellent electrical and thermal performances. However, under high dc electric field space charges can accumulate inside the silicone rubber, which will distort the electric field in the bulk of the material, influencing the reliability and safety of operation. A bipolar charge injection and transport model is adopted to investigate the accumulation of space charges and distortion of electric field in silicone rubber. It is found that when charge injection rate is higher than the charge migration rate, space charges will accumulate in the material and the accumulation increases with an increase in electric field. The influence of the Poole-Frenkel effect is then studied. It is found that stronger Poole-Frenkel effect can enhance the nonlinearity of effective carrier mobility of silicone rubber, reducing the accumulation of space charges. This indicates that tuning the nonlinearity of effective carrier mobility by nano-doping can suppress space charges, improving the reliability of power cable accessories.
\end{abstract}

\section{Introduction}

Due to the rapid development of the economy, a surging demand for electricity is rising in China. By the end of 2017, State Grid Corporation of China has 1.38 TW of power generating capacity in its service area, growing by $8.5 \%$ compared to the previous year and accounting for $78 \%$ of the total installed generating capacity in China [1]. In addition, the electrical energy consumptions in metropolises are very high, which are $106 \mathrm{G} \mathrm{kWh}$ and $152 \mathrm{G} \mathrm{kWh}$ in Beijing and Shanghai, respectively [2]. The rapid increase of electrical energy consumption in metropolises requires more power cables to be utilized in power grid or urban power network to transmit and distribute electrical energy [3].

Recent years, high voltage direct current (HVDC) power cables are widely used in power grid. Accordingly, the quantity demanded for power cable accessories which are used to interconnect two power cables or a power cable and a power apparatus increases rapidly. Power cable accessories can mitigate the electric field distortion at the cutting point of shielding layer, avoiding the occurrence of electrical breakdown or surface flashover on power cables. However, power cable accessories operate in a harsh environment consisting of distorted electric field, high temperature, and mechanical stress, which can result in fault with high probability. Considering the complexity of cable structure and distribution of $\mathrm{dc}$ electric field, the problem is more

a Corresponding author: forrestmin @xjtu.deu.cn severely for dc power cable accessories. Statistical results show that more than $70 \%$ failures in power cable transmission lines are caused by the faults of power cable accessories [3, 4]. Therefore, as an important component of dc power cable transmission system, the operation safety of power cable accessories is a key issue for the reliability of power system.

For HVDC power cable, the electric field is distributed according to dc conductivity in polymeric insulating materials. Since the $\mathrm{dc}$ conductivity of polymeric insulating materials depends strongly on electric field and temperature. The electric field is nonuniform in polymeric insulating materials. Silicone rubber is widely used as polymeric insulating materials in power cable accessories due to their excellent electrical and thermal performances. It is of interest to investigate the space charge formation and accumulation properties in silicone rubber under high dc electric field via a bipolar charge injection and transport model.

\section{Bipolar charge injection and transport model}

Figure 1 illustrates the schematic of bipolar charge injection and transport model in a polymeric material [5, 6]. A polymeric material with a thickness of $d$ is clamped by two electrodes, and a voltage is applied to the material. Electrons and holes are injected into the conduction band 
$E_{C}$ and valence band $E_{V}$ of the polymeric material. The injected electrons and holes will migrate toward anode and cathode under the applied electric field. There are a lot of traps in polymeric materials formed by physical disorders and chemical impurities. These traps can capture mobile electrons and mobile holes during their migration. Trapped charges can be activated to mobile states by thermal energy or Poole-Frenkel effect which means the potential barrier can be reduced by an electric field to assist the detrapping of charges [7-9]. When mobile electrons and mobile holes encounter each other in the material, they will recombine via Shockley-ReadHall mechanism [10]. While mobile electrons and trapped holes, as well as mobile holes and trapped electrons will recombine via Langevin recombination mechanism [11]. Electrons and holes that are not recombine inside the material will flow out to its electrodes.

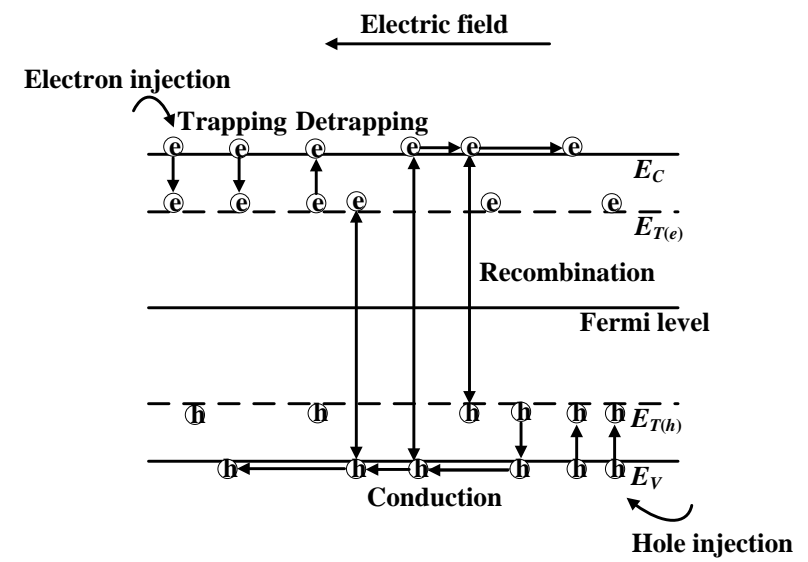

Figure 1. Schematic diagram of bipolar charge injection and transport model in a polymeric material. Here $E_{C}$ and $E_{V}$ are the energies of conduction band and valence band respectively, and $E_{T(e)}$ and $E_{T(h)}$ are the energies of deep traps for electrons and holes respectively in the forbidden band.

\subsection{Injections of positive and negative charges from electrodes into silicone rubber}

The injected current densities of electrons and holes from anode and cathode, $j_{i n(e)}$ and $j_{i n(h)}$, are determined by electric field, temperature, and effective potential barrier, which are described by following equations [12],

$$
\begin{gathered}
j_{i n(e)}=4 \psi^{2} N_{C} e \mu_{C} F \exp \left(-E_{T(e)} / k_{B} T\right) \exp f^{1 / 2} \\
j_{i n(h)}=4 \psi^{2} N_{V} e \mu_{V} F \exp \left(-E_{T(h)} / k_{B} T\right) \exp f^{1 / 2}
\end{gathered}
$$

where $N_{C}$ and $N_{V}$ are the densities of states in conduction band and valence band in $1 / \mathrm{m}^{3}$ respectively, $e$ is the elementary charge in $\mathrm{C}, \mu_{C}$ and $\mu_{V}$ are carrier mobilities in conduction band and valence band in $\mathrm{m}^{2} /(\mathrm{Vs})$ respectively, $F$ is the electric field in V/m, $E_{T(e)}$ and $E_{T(h)}$ are the energies of deep traps for electrons and holes in $\mathrm{eV}, k_{B}$ is the Boltzmann constant, and $T$ is the sample temperature in K. Furthermore, $f$ is the reduced electric field and $\psi$ is the field-dependent parameter. The carrier mobilities in conduction band and valence band are assumed to be $1 \times 10^{-5} \mathrm{~m}^{2} /(\mathrm{Vs})$.

The reduced electric field is determined by Coulomb radius, electric field, and temperature, which is $f=e F r_{c} / k_{B} T$. The coulomb radius $r_{c}$ is equal to $e^{2} / 4 \pi \varepsilon_{0} \varepsilon_{r} k_{B} T$.
Here $\varepsilon_{0}$ is the permittivity of vacuum in $\mathrm{F} / \mathrm{m}$ and $\varepsilon_{r}$ is the dielectric constant of silicone rubber. Then, the fielddependent parameter $\psi(f)$ can be obtained by the following equation $\psi(f)=f^{-1}+f^{1 / 2}-f^{-1}\left(1+2 f^{1 / 2}\right)^{1 / 2}$.

\subsection{Self-consistent drift-diffusion equations}

A set of self-consistent drift-diffusion equations are utilized to calculate the transport and accumulation of space charges and the distribution of electric field inside silicone rubber, and the conduction current through the material. The self-consistent drift-diffusion equations consist of charge conduction equation, charge continuity equation, and Poisson's equation $[5,6]$.

$$
\begin{gathered}
j_{c(e)}(x, t)=q_{\text {free }(e)}(x, t) \mu_{0(e)} F(x, t) \\
j_{c(h)}(x, t)=q_{\text {free }(h)}(x, t) \mu_{0(h)} F(x, t) \\
\frac{\partial q_{\text {free }(e)}(x, t)}{\partial t}+\frac{\partial q_{\text {trap }(e)}(x, t)}{\partial t}+\frac{\partial j_{c(e)}(x, t)}{\partial t}=S_{e} \\
\frac{\partial q_{\text {free }(h)}(x, t)}{\partial t}+\frac{\partial q_{\text {trap }(h)}(x, t)}{\partial t}+\frac{\partial j_{c(h)}(x, t)}{\partial t}=S_{h} \\
\frac{\partial^{2} \phi(x, t)}{\partial x^{2}}=-\frac{q_{n e t}(x, t)}{\varepsilon_{0} \varepsilon_{r}}
\end{gathered}
$$

where $j_{c(e)}$ and $j_{c(h)}$ are the conduction current densities of electrons and holes in conduction band and valence band in $\mathrm{A} / \mathrm{m}^{2}$ respectively, $q_{\text {free }(e)}$ and $q_{\text {free }(h)}$ are the carrier densities of electrons and holes in $\mathrm{C} / \mathrm{m}^{3}$ respectively, $q_{\text {trap }(e)}$ and $q_{\text {trap }(h)}$ are the densities of trapped electrons and trapped holes in $\mathrm{C} / \mathrm{m}^{3}$ respectively, $\mu_{0(e)}$ and $\mu_{0(h)}$ are the carrier mobilities of electrons and holes in silicone rubber in $\mathrm{m}^{2} /(\mathrm{Vs})$ respectively, $S_{e}$ and $S_{h}$ are the charge trappingdetrapping-recombination terms for electrons and holes respectively, $\phi$ is the potential in the material in $\mathrm{V}$, and $q_{\text {net }}$ is the net charge density, which is $q_{\text {net }}=q_{\text {free }(e)}+q_{\text {trap }(e)}+$ $q_{\text {free }(h)}+q_{\text {trap }(h)}$. Furthermore, $x$ is the position in the material and $t$ is the elapse time after the application of voltage on the material.

\subsection{Charge trapping-detrapping-recombination dynamic equations}

The trapping-detrapping-recombination dynamics for mobile electrons $S_{e \mu}$, trapped electrons $S_{e t}$, mobile holes $S_{h \mu}$, and trapped holes $S_{h t}$ are described by the following equations $[5,6]$.

$$
\begin{aligned}
& S_{e \mu}=-P_{\operatorname{tr}(e)} q_{\text {free }(e)}\left(1-q_{\operatorname{trap}(e)} / e N_{T(e)}\right)+P_{d e(e)} q_{\operatorname{trap}(e)} \\
& -R_{e \mu, h \mu} q_{\text {free }(e)} q_{\text {free }(h)}-R_{e \mu, h t} q_{\text {free }(e)} q_{\text {free }(h)} \\
& S_{e t}=P_{t r(e)} q_{\text {free }(e)}\left(1-q_{\operatorname{trap}(e)} / e N_{T(e)}\right)-P_{d e(e)} q_{\operatorname{trap}(e)} \\
& -R_{e t, h \mu} q_{\text {trap }(e)} q_{\text {free }(h)} \\
& S_{h \mu}=-P_{\text {tr }(h)} q_{\text {free }(h)}\left(1-q_{\text {trap }(h)} / e N_{T(h)}\right)+P_{\text {de }(h)} q_{\text {trap }(h)} \\
& -R_{e \mu, h \mu} q_{\text {free }(e)} q_{\text {free( } h)}-R_{e t, h \mu} q_{\text {trap }(e)} q_{\text {free }(h)} \\
& S_{h t}=P_{t r(h)} q_{\text {free }(h)}\left(1-q_{\text {trap }(h)} / e N_{T(h)}\right)-P_{d e(h)} q_{\text {trap }(h)} \\
& -R_{e \mu, h t} q_{\text {free }(e)} q_{\text {trap }(h)}
\end{aligned}
$$


where $P_{\operatorname{tr}(e)}$ and $P_{\operatorname{tr}(h)}$ are the trapping coefficients for mobile electrons and holes in $\mathrm{s}^{-1}$ respectively and $P_{t r(e, h)}=e N_{T(e, h)} / \varepsilon 0 \varepsilon r, N_{T(e)}$ and $N_{T(h)}$ are the densities of deep traps for electrons and holes in $1 / \mathrm{m}^{3}$ respectively, $R_{e \mu, h \mu}$ is the recombination coefficient between mobile electrons and mobile holes, $R_{e \mu, h \mu}=\left(\mu_{0(e)}+\mu_{0(h)}\right) / \varepsilon_{0} \varepsilon_{r}$. $R_{e \mu, h t}$ and $R_{e t, h \mu}$ are the trap-assisted recombination coefficients between mobile electrons and trapped holes and between trapped electrons and mobile holes respectively, $R_{e \mu, h t}=\mu_{0(e)} / \varepsilon_{0} \varepsilon_{r}$ and $R_{e t, h \mu}=\mu_{0(h)} / \varepsilon_{0} \varepsilon_{r}$.

The charge detrapping coefficients $P_{d e}$ is described by the following equation $[5,6]$.

$$
P_{d e}=v_{0} \exp \left(-\frac{E_{T}}{k_{B} T}\right) G(F)
$$

where $v_{0}$ is the attempt-to-escape frequency in $\mathrm{s}^{-1}$, which is assumed to be $1 \times 10^{12} \mathrm{~s}^{-1}$, and $G(F)$ is the PooleFrenkel effect improved by Ieda et al [7].

$$
\begin{gathered}
G(F)=\frac{4 \gamma}{\alpha^{2} F} \sinh \left(\frac{\alpha^{2} F}{4 \gamma}\right), \alpha^{2} F \leq 2 \gamma \\
G(F)=\frac{1}{\alpha^{2} F}\left[\left(\alpha F^{1 / 2}-1\right) \exp \left(\alpha F^{1 / 2}-\gamma\right)+\exp (\gamma)\right] \\
-\frac{2 \gamma}{\alpha^{2} F} \exp \left(-\frac{\alpha^{2} F}{4 \gamma}\right), \alpha^{2} F>2 \gamma
\end{gathered}
$$

where $\alpha$ is a coefficient relating to the potential barrier lowering caused by Pool-Frenkel effect, $\alpha=$ $2\left(e^{3} / 4 \pi \varepsilon_{0} \varepsilon_{r}\right)^{1 / 2} /\left(m k_{B} T\right)$, and $m$ is constant changing from 1 to 2 , while $\gamma$ is a constant which is $3 / 2$ in the present work.

\subsection{Charge transport parameters for silicone rubber}

The dielectric constant measured by novocontrol broadband dielectric relaxation spectroscopy is about 3.5 at $50 \mathrm{~Hz}$ at room temperature. The carrier mobility controlled by shallow traps is $7.0 \times 10^{-14} \mathrm{~m}^{2} /(\mathrm{Vs})$ for both electrons and holes [13]. The densities of deep traps for electrons and holes are $6.2 \times 10^{20} 1 / \mathrm{m}^{3}$ according to surface potential decay experiments, while the energies of deep traps for electrons and holes are $0.90 \mathrm{eV}$ [13]. The potential barriers are assumed to be the same as deep traps for the charge injections from cathode and anode into silicone rubber.

\section{Simulation results and discussion}

\subsection{Distributions of space charges and electric fields}

Figure 2 shows space charge distributions in silicone rubber at various times under an applied voltage of $50 \mathrm{kV}$ at constant $m$ of 2.0. After the application of voltage, electrons and holes will be injected into the polymeric material by equations (1) and (2) respectively. Since the carrier mobility of the silicone rubber is very low and deep traps in the material can capture mobile charge carriers, injected charges can accumulate in the bulk, which is called homo space charges. As shown in Figure 2 , negative charges are accumulated in the area near the cathode, while positive charges are accumulated in the area near the anode. As electrons and holes move toward anode and cathode under electric field respectively, the magnitude and area of space charges increase with time. The magnitudes of positive space charges are $7.27 \mathrm{C} / \mathrm{m}^{3}$ and $53.68 \mathrm{C} / \mathrm{m}^{3}$ at $t$ of $10 \mathrm{~s}$ and $1000 \mathrm{~s}$, respectively. The widths of space charge accumulation are $0.027 \mathrm{~mm}$ and $0.265 \mathrm{~mm}$ at $t$ of $10 \mathrm{~s}$ and $1000 \mathrm{~s}$, respectively.

Figure 3 shows the distributions of electric fields in silicone rubber at various times under an applied voltage of $50 \mathrm{kV}$ at constant $m$ of 2.0. As homo space charges accumulating in silicone rubber, electric fields near the interfaces between the material and its electrodes decreases gradually, while electric fields in the middle of the material increase with time. The electric fields at the interface between the silicone rubber and cathode are reduced from $5.00 \times 10^{7} \mathrm{~V} / \mathrm{m}$ to $3.64 \times 10^{7} \mathrm{~V} / \mathrm{m}$ and $7.93 \times 10^{6} \mathrm{~V} / \mathrm{m}$ at $50 \mathrm{~s}$ and $1000 \mathrm{~s}$, respectively. The electric fields in the middle of the material are $5.05 \times 10^{7}$ $\mathrm{V} / \mathrm{m}$ and $5.44 \times 10^{6} \mathrm{~V} / \mathrm{m}$ at $50 \mathrm{~s}$ and $1000 \mathrm{~s}$, respectively.

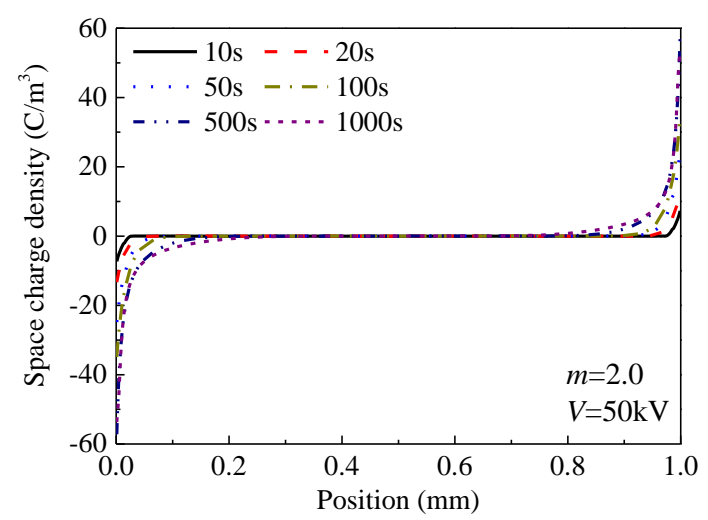

Figure 2. Distributions of space charges in silicone rubber at various times at $m$ of 2.0 .

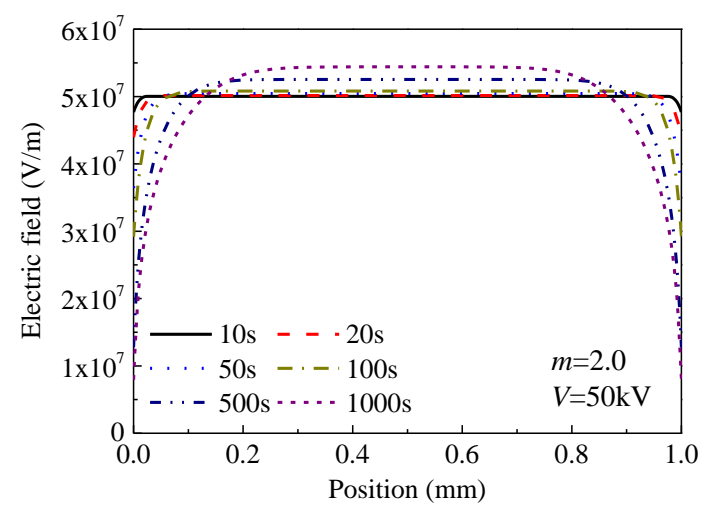

Figure 3. Distributions of electric fields in silicone rubber at various times at $m$ of 2.0 .

Figure 4 demonstrates space charge distributions at steady state in silicone rubber at various electric fields at $m$ of 2.0. The higher electric field can result in more electrons and holes being injected into the polymeric material. The magnitudes of positive space charges are $30.92 \mathrm{C} / \mathrm{m}^{3}$ and $61.06 \mathrm{C} / \mathrm{m}^{3}$ at electric fields of $1 \times 10^{7} \mathrm{~V} / \mathrm{m}$ and $9 \times 10^{7} \mathrm{~V} / \mathrm{m}$, respectively. The area accumulated space charges is also increased by increasing the electric field. The widths of space charge accumulation are $0.026 \mathrm{~mm}$ 
and $0.447 \mathrm{~mm}$ at electric fields of $1 \times 10^{7} \mathrm{~V} / \mathrm{m}$ and $9 \times 10^{7} \mathrm{~V} / \mathrm{m}$, respectively.

Figure 5 shows the electric field distributions at steady state in silicone rubber at various electric fields at $m$ of 2.0. More homo space charges are injected and accumulated inside the polymeric material can cause severer distortion of electric field. The maximum electric fields in the middle of silicone rubber are $1.01 \times 10^{7} \mathrm{~V} / \mathrm{m}$ and $1.01 \times 10^{8} \mathrm{~V} / \mathrm{m}$ at electric fields of $1 \times 10^{7} \mathrm{~V} / \mathrm{m}$ and $9 \times 10^{7} \mathrm{~V} / \mathrm{m}$, respectively.

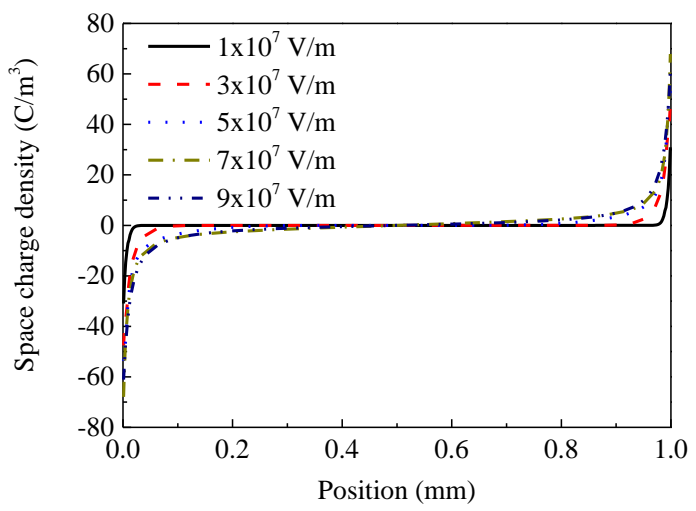

Figure 4. Space charge distributions of silicone rubber at various electric fields at $m$ of 2.0 .

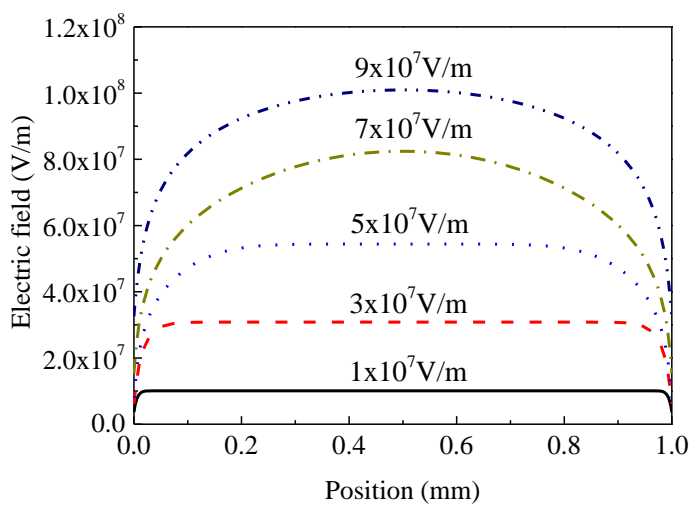

Figure 5. Electric field distributions of silicone rubber at various electric fields at $m$ of 2.0 .

\subsection{Electric field dependent current density and space accumulation}

Under the application of electric field, carriers will transport through the material, forming current which can be detected from the external circuit by a pico-ampere meter. Figure 6 demonstrates current density flowed through silicone rubber as a function of electric field at various $m$. The potential barrier width of trapped charges can be modified by the Poole-Frenkel effect. Smaller $m$ means stronger Poole-Frenkel effect, which can facilitate the migration of charge carriers. It is observable that the current density is higher at smaller $m$.

The simulation results indicate that changing the constant $m$ by incorporating nanoparticles into polymeric materials can tune their nonlinear conductivity. It can maintain high resistivity at relatively low electric fields. At high electric fields, it then can facilitate the migration of charge carriers so that reduce the accumulation of space charges, which is shown in Figure 7. It indicates that tuning the nonlinearity of effective carrier mobility can suppress the accumulation of space charges, improving the reliability of power cable accessories.

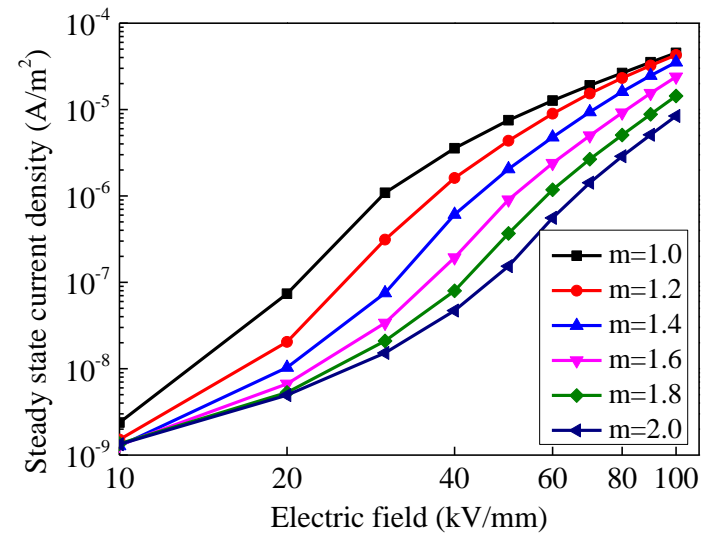

Figure 6. Current density as a function of electric field in silicone rubber at various $m$ from 1.0 to 2.0.

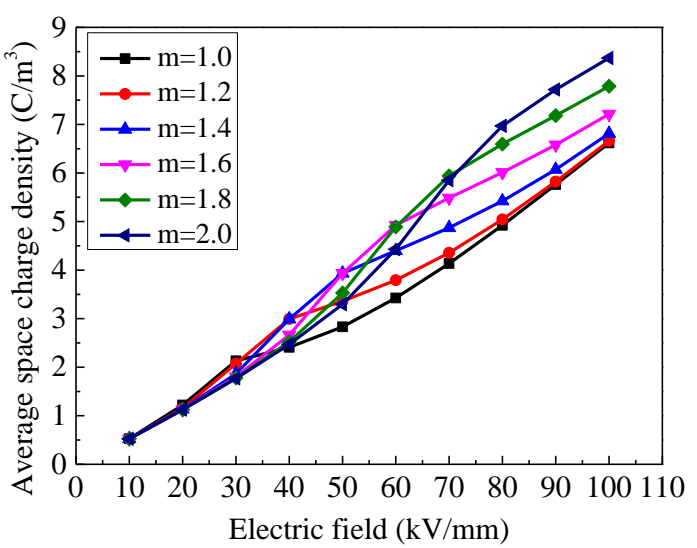

Figure 7. Average space charge accumulation as a function of electric field in silicone rubber at various $m$ from 1.0 to 2.0.

\section{Conclusion}

A model consisting of bipolar charge injection and transport as well as charge trapping-detrappingrecombination dynamics with Poole-Frenkel effect is adopted to investigate the accumulation of space charges and distortion of electric field in silicone rubber. It was found space charges will accumulate in the material when charge injection rate is higher than the charge migration rate, and the accumulation increases with the increase in electric field. Then, we studied the influence of PooleFrenkel effect on space charge accumulation and external current density. It was found that stronger Poole-Frenkel effect with smaller $m$ can enhance the nonlinearity of effective carrier mobility of silicone rubber, reducing the accumulation of space charges, which can improve the operational safety of power cable accessories.

\section{References}

This work is supported by the State Grid Corporation of China Science Projects "Study on the Influence of Harmonic Voltage on Cable Joint and Capacitive 
Bushing" (EPRIGYKJ(2015)4268) and "Study on computational method of electric field in dc insulation system" ( no. 522722160001).

\section{References}

[1]http://www.sgcc.com.cn/html/sgcc main/col20170412 41/column_2017041241_1.shtml and https:// chinaenergy portal.org /2017-electric-power-industry -summary-statistics/.

[2]http://shoudian.bjx.com.cn/html/20180129/877269.sht $\mathrm{ml}$ and https://baijiahao.baidu.com/s?id=1594623958 $914905384 \& w f r=$ spider \&for $=p c$.

[3] Y. Zhou, S. M. Peng, J. Hu and J. L. He, IEEE Trans. Dielectr. Electr. Insul., 24, 1308-1318, (2017)

[4] Y. J. Fei, Y. X. Zhang and Y. X. Zhou, Adv. Tech. Electr. Eng. Ener., 33, 30-34, (2014)

[5] D. M. Min, C. Y. Yan, R. Mi, H. Z. Cui, Y. W. Li, W.
W. Wang, M. Frechette and S. T. Li, IEEE Nanotechnology Magazine, 2, 15-22, (2018)

[6] D. M. Min, S. T. Li and Y. Ohki, IEEE Trans. Dielectr. Electr. Insul., 23, 507-516, (2016)

[7] M. Ieda, G. Sawa and S. Kato, J. Appl. Phys., 42, 3737-3740, (1971)

[8] J. L. Hartke, J. Appl. Phys., 39, 4871-4873, (1968)

[9] J. G. Simmons, Phys. Rev., 155, 657-660, (1967)

[10] W. Shockley and W. T. Read, Phys. Rev., 87, 835$842,(1952)$

[11] J. J. M. van der Holst, F. W. A. van Oost, R. Coehoorn and P. A. Bobbert, Phys. Rev. B, 80, 235202(8), (2009)

[12] J. C. Scott and G. G. Malliaras, Chem. Phys. Lett., 299, 115-119, (1999)

[13] D. M. Min, C. Y. Yan, Y. Huang, S. T. Li and Y. Ohki, Polymers, 9, 1-15, (2017) 